\title{
EVALUACIÓN SISTÉMICA DE UNA POLÍTICA DE FLEXIBILIDAD DE VOLUMEN EN UNA CADENA DE SUMINISTRO DISTRIBUIDORA DE PAPAYA
}

\section{ANDRÉs Mauricio PAREdes RodríGuez ${ }^{1}$ ANDRÉS FELIPE SALAZAR RAMOS ${ }^{2}$}

\section{RESUMEN}

La flexibilidad de volumen es utilizada en el presente artículo para determinar la capacidad de respuesta ante cambios en los factores de deterioro de un producto perecedero en las etapas de una cadena de suministro. El objetivo principal de este estudio es evaluar el impacto que tiene esta estrategia sobre dos indicadores claves para el desempeño de la red como lo son los costos logísticos y el nivel de servicio. La investigación involucra el funcionamiento de una red de distribución de papaya conformada por un productor y un detallista, la cual es simulada a través de Dinámica de Sistemas. Se logra establecer que una política de flexibilidad de volumen mejora notablemente indicadores como el nivel de servicio y los costos considerados en el estudio; sin embargo, utilizar esta estrategia implica asumir un mayor costo de almacenamiento de inventario y un costo adicional de aumento de capacidad.

PALABRAS CLAVE: Capacidad, flexibilidad de volumen, productos perecederos, cadena de suministro, papaya, Dinámica de Sistemas.

\section{SYSTEMIC EVALUATION OF A POLICY OF VOLUME FLEXIBILITY IN A PAPAYA DISTRIBUTION SUPPLY CHAIN}

\section{ABSTRACT}

Volume flexibility is used in this article to determine responsiveness to changes in the deterioration factors of perishable stages in a supply chain. The main objective of this study is to evaluate the impact of this strategy on two key indicators for network performance, such as logistics costs and service levels. The research involves the operation of a papaya distribution network formed by a producer and a retailer, which is simulated through System Dynamics. A policy of flexibility is achieved, which dramatically improves volume indicators such as the level of service and costs considered in the study. However, using this strategy involves taking on a higher cost of inventory storage and an additional cost of capacity increase.

1 Ingeniería Industrial. Sociedad Ferretera de Comercio S.A. San Pedro, Valle, Colombia.

2 Maestría en Ingeniería con énfasis en Ingeniería Industrial. Universidad del Valle, Colombia.

Autor de correspondencia: Paredes Rodríguez, A.M. (Andrés Mauricio): Calle 3 \#2-34, San Pedro, Valle del Cauca, Colombia. Tel.: 3152142940. Correo electrónico: andres.paredes@correounivalle.edu.co
Historia del artículo:

Artículo recibido: 15-II-2016 / Aprobado: 26-VI-2017

Disponible online: 30 de agosto de 2017

Discusión abierta hasta octubre de 2018 
KEYWORDS: Capacity, Flexibility Volume, Perishables, Supply Chain, Papaya, System Dynamics.

\section{AVALIAÇÃO SISTÉMICA DE UMA POLÍTICA DE FLEXIBILIDADE DE VOLUME NUMA CORRENTE DE FORNECIMENTO REVENDEDORA DE MAMÃO}

\section{RESUMO}

A flexibilidade de volume é utilizada no presente artigo para determinar a capacidade de resposta ante mudanças nos fatores de deterioração de um produto perecível nas etapas de uma corrente de fornecimento; o objetivo principal deste estudo é avaliar o impacto que tem esta estratégia sobre dois indicadores chaves para o desempenho da rede como o são os custos logísticos e o nível de serviço. A investigação envolve o funcionamento de uma rede de distribuição de mamão conformada por um produtor e um detalhista, a qual é simulada através de Dinâmica de Sistemas. Consegue-se estabelecer que uma política de flexibilidade de volume melhora notavelmente indicadores como o nível de serviço e os custos conceituados no estudo; no entanto, utilizar esta estratégia implica assumir um maior custo de armazenamento de inventário e um custo adicional de aumento de capacidade.

PALAVRAS-CHAVE: Capacidade, flexibilidade de volume, produtos perecíveis; corrente de fornecimento; mamão; sistemas dinâmicos.

\section{INTRODUCCIÓN}

La cadena de suministro se puede definir como toda la cadena de valor del producto, desde el abastecimiento de la materia prima hasta la adquisición por parte del cliente final (Croom, Romano, \& Giannakis, 2000; Jack \& Raturi, 2002). En la actualidad los cortos ciclos de vida de los productos, la competencia más intensa y el aumento de las expectativas del cliente, ha provocado una mayor complejidad en la gestión de cadenas de suministro (Blome, Schoenherr, \& Eckstein, 2014). El aumento de la incertidumbre y el riesgo en las cadenas de suministro globales añade otro nivel de complejidad a su gestión (Blome, Schoenherr, \& Eckstein, 2014). Por lo tanto, es necesario establecer estructuras que conduzcan a una generalización de flexibilidad, es decir, acercarse a marcos que mejoren la capacidad de una empresa para adaptarse o responder a los cambios (Braunscheidel \& Suresh, 2009)
En el siglo pasado, la flexibilidad estaba enfocada solo hacia operaciones de manufactura; sin embargo, debido al aumento de la globalización, la tendencia a la subcontratación y la elevada complejidad del ambiente, hoy en día la investigación relativa a la flexibilidad se ha ampliado a las cadenas de suministro (Blome et al., 2014).Sin embargo, gran parte de la investigación existente tiene una definición limitada de flexibilidad de la cadena de suministro y describen la flexibilidad simplemente como un medio para hacer frente a la incertidumbre (Stevenson \& Spring, 2007). Por este motivo algunos autores como Holweg (2005) y Kumar, Fantazy, Kumar, \& Boyle (2006) exponen que existe una urgente necesidad para llevar a cabo nuevas investigaciones empíricas en flexibilidad de cadena de suministro.

La flexibilidad asociada a la gestión de cadenas de suministro de productos perecederos se considera compleja debido al alto nivel de incertidumbre que se genera por la inexactitud de las cantidades, 
tiempos y especificaciones de la demanda del cliente final (Disney \& Towill, 2003; Lee, Padmanabhan, \& Whang, 1997), esto conlleva a que sea necesario asegurar que el detallista tenga un mayor nivel de inventario a la mano para ser capaz de absorber las fluctuaciones de la demanda (Stevenson \& Spring, 2007) y de esta forma ofrecer un alto nivel de servicio, es decir, tener una baja probabilidad de presentar escenarios de venta pérdida o backorder ${ }^{1}$.

Al ser la flexibilidad una herramienta para reducir la incertidumbre en la demanda (Blome, Schoenherr, \& Eckstein, 2014) se convierte en base fundamental para mejorar la gestión de una cadena de suministro de productos perecederos, ya que al tener una buena planificación de la demanda, se controlan problemáticas indeseables en el sistema, tales como el bajo nivel de servicio y los elevados costos logísticos asociados al mantenimiento de este tipo de producto. Por este motivo en el caso de estudio, se abordará el problema de flexibilidad en una cadena de suministro que distribuye Papaya a través de la perspectiva de un incremento de flexibilidad de volumen.

Para realizar la caracterización de flexibilidad de volumen en la cadena de suministro de la Papaya, se realizó una revisión literaria sobre este concepto usando como criterios de búsqueda, palabras clave como flexibilidad, aumento de capacidad, adaptabilidad y agilidad. En la anexo 1 se presenta una tabla resumen con aquellos aspectos más relevantes encontrados dentro de la revisión.

Un aspecto clave a resaltar dentro de la búsqueda literaria es el hecho de que la flexiblidad en cadenas de suministro ha venido experimentado un creciente interés en la academia, ya que cada vez existe un mayor número de artículos que abordan este tipo de problemática desde distintos puntos de vista, hecho reflejado especialmente en los años 2009 y 2011 en donde se presentó un mayor número de publicaciones sobre la temática (ver Gráfico 1).

1 Pedido pendiente que se adquiere cuando el cliente puede aceptar que su orden completa sea clasificada como requisición pendiente (Vidal, 2010).

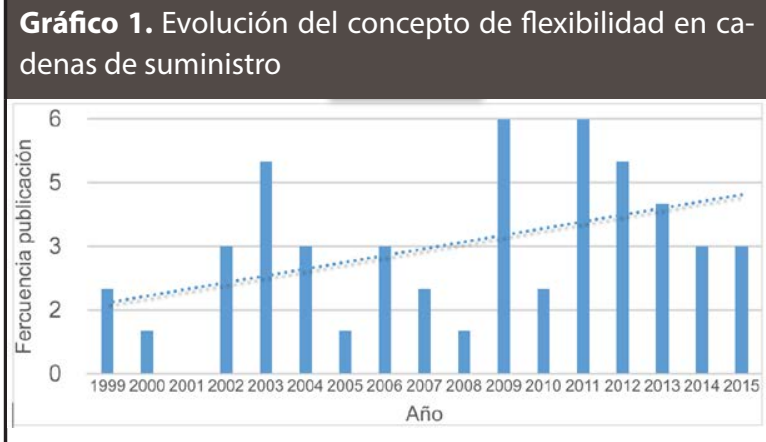

Uno de los grandes aportes logrado gracias a la revisión literaria es la definición de los tipos de flexibilidad existentes, los cuales según Vickery, Calantone, \& Dröge (1999) son: Flexibilidad de producto, volumen, nuevo producto, distribución y respuesta. En esta investigación se utilizará la flexibilidad de volumen debido a que Vickery et al (1999)

Lograron establecer la importancia de este tipo de flexibilidad en el cumplimiento de las demandas de los clientes y en el mejoramiento de la capacidad de respuesta, es decir, que si una empresa consigue crear un sistema de flexibilidad de volumen tenderá a tener niveles de servicio más altos. Además Zhang (2003) logra establecer que este tipo de flexibilidad tiene una relación fuertemente positiva con la satisfacción del cliente final.

La razón por la que se selecciona la Papaya para este caso de investigación es que un estudio realizado por la Universidad Nacional concluyó que esta fruta es uno de los productos que puede generar iniciativas que tengan un amplio potencial para la generación de ingresos de las personas en la zona Norte del Valle del Cauca (Castellano et al., 2011), lo que puede promover el crecimiento económico de la región.

El documento se desarrolla de la siguiente manera: en la sección 2 se describe la metodología con la que se abordó el problema objeto de estudio, en la sección 3 se muestran los resultados más representativos del modelo, realizando un análisis de sensibilidad de algunos parámetros y exhibiendo unos escenarios alternos al inicialmente planteado, en la sección 4 se presenta una discusión de los resultados 
y por último en la sección 5 se exhiben las conclusiones particulares obtenidas luego de la investigación.

\section{METOdOLOGÍA}

La dinámica de sistemas fue desarrollada por Jay Wright Forrester en el año de 1961 en el Massachusetts Institute of Tecnology (MIT) con el objetivo de estudiar, modelar y simular el comportamiento de sistemas socio-económicos complejos. Desde entonces, la dinámica de sistemas se ha convertido en una herramienta eficaz para el análisis de todo tipo de políticas dentro de cualquier industria. - Ver: Forrester (1961); Senge (2006) - .

El presente estudio abordará el análisis de flexibilidad de volumen de una cadena de suministro de la papaya a través de dinámica de sistemas, debido a que esta herramienta permite visualizar claramente el flujo de información de los pedidos del detallista al fabricante, junto con sus respectivas demoras; permitiendo de esta forma establecer la estructura del sistema, con el objetivo de controlar comportamientos indeseables en la cadena de suministro como el desabastecimiento del detallista o la pérdida de confiabilidad del productor. Tako y Robinson (2012) han logrado establecer las ventajas de la dinámica de sistemas sobre otro tipo de herramientas como la simulación de eventos discretos, las cuales son:

- Toma un enfoque holístico de los sistemas, al integrar muchos subsistemas.

- Se centra en las políticas y la estructura del sistema.

- Usa bucles de retroalimentación para representar los efectos de las decisiones de la política.

- Representa una visión dinámica de las relaciones de causa y efecto entre los elementos del sistema.

- Tiene unos requisitos mínimos de datos para construir un modelo.

Existen estudios relevantes donde se puede evidenciar la aplicación de la dinámica de sistemas aplicados a productos perecederos o de muy corto ciclo de vida. Minegishi y Thiel (2000) buscan mediante la dinámica de sistemas analizar el control y la gestión de una cadena de suministro de alimentos, buscando contribuir al entendimiento del complejo comportamiento logístico de una cadena de suministro de aves de corral, haciendo especial énfasis sobre los tiempos de distribución necesarios en la cadena y cómo las actividades de planeación correspondientes deben alinearse para enfrentar los ciclos de demanda de estos productos. Higuchi y Troutt (2004) simulan mediante dinámica de sistemas, la cadena de suministro del producto Tamagoshi conformada por un fabricante, un mercado y un detallista; con el objetivo de ayudar toma de decisiones en cada uno de los eslabones del sistema.

Para este estudio se utilizaron aportes encontrados en una investigación previa realizada por los autores, en donde se define y analiza la flexibilidad de volumen en cadenas de suministro de productos perecederos (Paredes \& Salazar, 2014), pero de una forma general y no específica, como lo es en este caso, al utilizarse un caso de estudio aplicado al cultivo de la papaya.

El primer paso del proceso metodológico que plantea la Dinámica de sistemas consiste en la creación de un diagrama que relacione las principales variables y su influencia sobre las demás, a este esquematización se le conoce como diagrama causal. El diagrama causal de este trabajo estará basado en el construido previamente por Paredes \& Salazar (2014), el cual será modificado para adaptarse al caso particular de la cadena de suministro de la papaya.

Una descripción del funcionamiento de la cadena de suministro de la Papaya se puede observar en la Figura 1. El punto marcado como 1 en la imagen, representa la cosecha de la papaya, se debe aclarar que el tiempo en el que mantiene este producto en la plantación varía dependiendo del tipo de Papaya que se ha cultivado. Además la producción de Papaya se ve afectada por el número de hectáreas que se cultivan y el rendimiento por tonelada que tenga la zona en que ha sido sembrada. Luego de 
cumplir su vida fisiológica, la Papaya es retirada de la planta y llevada a un almacenamiento del proveedor (representado en la imagen por el punto 2) para que termine su ciclo de maduración. Cuando la Papaya ya se encuentra en óptimas condiciones para ser consumida por el cliente, esta es transportada en vehículos refrigerados (punto 3), buscando conservar la calidad del producto por el mayor tiempo posible. El producto es llevado al detallista (punto 4) el cual le provee un almacenamiento al producto, para luego venderlo al cliente final (punto 5).

En el diagrama causal se involucra la interacción entre un productor, un detallista y el cliente final de la cadena de suministro de la Papaya. El diagrama causal completo se presenta en la Figura 2. Se puede evidenciar los bucles de compensación que se generan en el sistema y los cuales son representados por balanzas. Uno de los elementos de especial interés en este trabajo es la característica pe- recedera del producto que se distribuye y la cual es señalada en el diagrama causal como tres variables externas al sistema, el factor de desperdicio del detallista (asociado al almacenamiento del producto) y el factor de desperdicio del productor (asociado al almacenamiento del producto y al transporte y su tiempo de entrega o "Lead Time"). En cuanto al análisis de esta tasa de desperdicio, en la revisión realizada por Li et al. (2010) se determina que hay algunos documentos de investigación de productos perecederos que la consideran constante (ver: Padmanabhan y Vrat (1995), Bhunia y Maiti (1999)) y otros representan esta tasa como una función dependiente del tiempo con cierta distribución aleatoria (ver: Nita.H. Shah (1993), Sicilia, González, Febles, y Alcaide (2014). Para el caso de estudio realizado en el presente trabajo se considerará el análisis con los tres tipos de factores señalados constantes a través del tiempo.

Figura 1. Descripción de la cadena de suministro de la Papaya
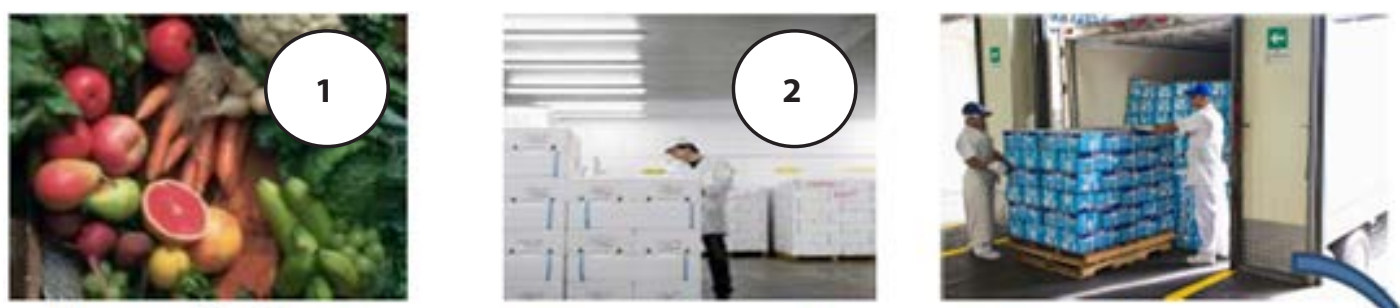

Almacenes frigorificos Situados en las zonas Productoras

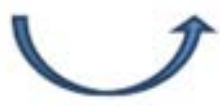

Almacenes Frigorificos

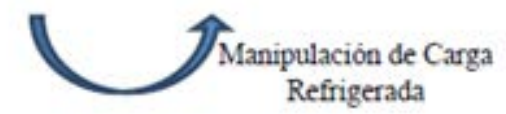

Refrigerada
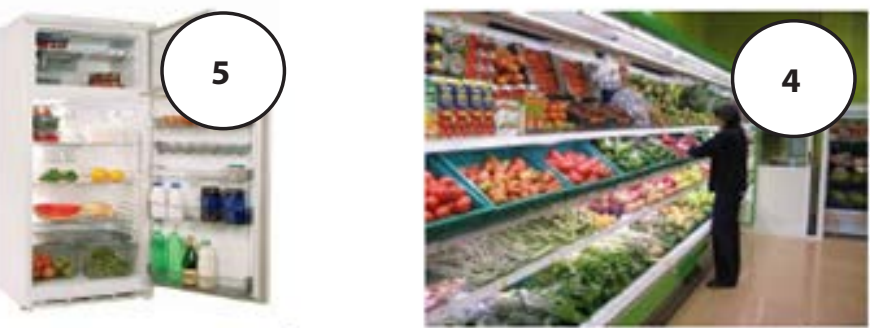

Consumidor Final

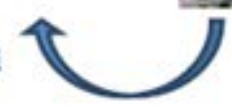

Supermercados

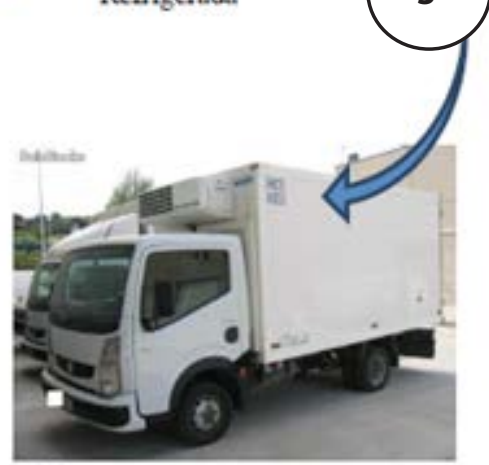

Vehiculos de Transporte

Frigorificos 
En la Figura 2, se puede notar como el productor comienza con una capacidad inicial, la cual afecta directamente su cosecha; sin embargo es necesario aclarar que existe una demora entre el momento en que se siembra y se recoge la cosecha. Luego de que la Papaya termina su ciclo de maduración en el inventario del productor, esta es enviada al detallista, aunque como ya se explicó, un porcentaje de la Papaya que se ha cosechado se degrada en el inventario del productor y por ende no puede ser enviada al minorista.

En el diagrama causal también se puede evidenciar como la demanda del cliente final afecta directamente al inventario del detallista, ya que al presentarse valores de demanda elevados es necesario acumular un nivel alto de inventario para responder satisfactoriamente a las necesidades del cliente, sin embargo, ésta puede ser una medida muy arriesgada porque existe un factor de desperdicio asociado a mantener por un tiempo prolongado, productos que tienen una vida útil corta como lo son los perecederos.

A medida que se comienza a agotar el inventario que el detallista tiene disponible, este agente se verá en la necesidad de emitir órdenes al productor; pero a su vez debe tener en cuenta que debido a la merma natural que presentan los productos perecederos se debe pedir más de lo que se necesita, para lograr satisfacer el requisito del cliente, a pesar de que una proporción del pedido se degrada y pierde en el recorrido. Entre mayor sea las órdenes atrasadas que el detallista vaya acumulando, la percepción de nivel de servicio por parte del cliente será menor, lo que ocasionará que el cliente deje de demandarle el producto a dicho minorista.

$\mathrm{Al}$ incrementar el tamaño y la frecuencia de los pedidos, se ocasiona un embotellamiento de las órdenes y algunos pedidos comienzan a retrasarse ya que el productor puede que no tenga la suficiente capacidad para cumplir con la cantidad de productos que solicita el detallista. Por este motivo la política de inversión de incremento de capacidad estará orientada a la adquisición de hectáreas para la siembra del cultivo de Papaya. Con el incremento en la capacidad del productor lo que se busca es lograr mejorar el nivel de servicio al cliente mediante una cadena de suministro flexible.

\section{Figura 2. Diagrama causal de la cadena de suministro de la Papaya}

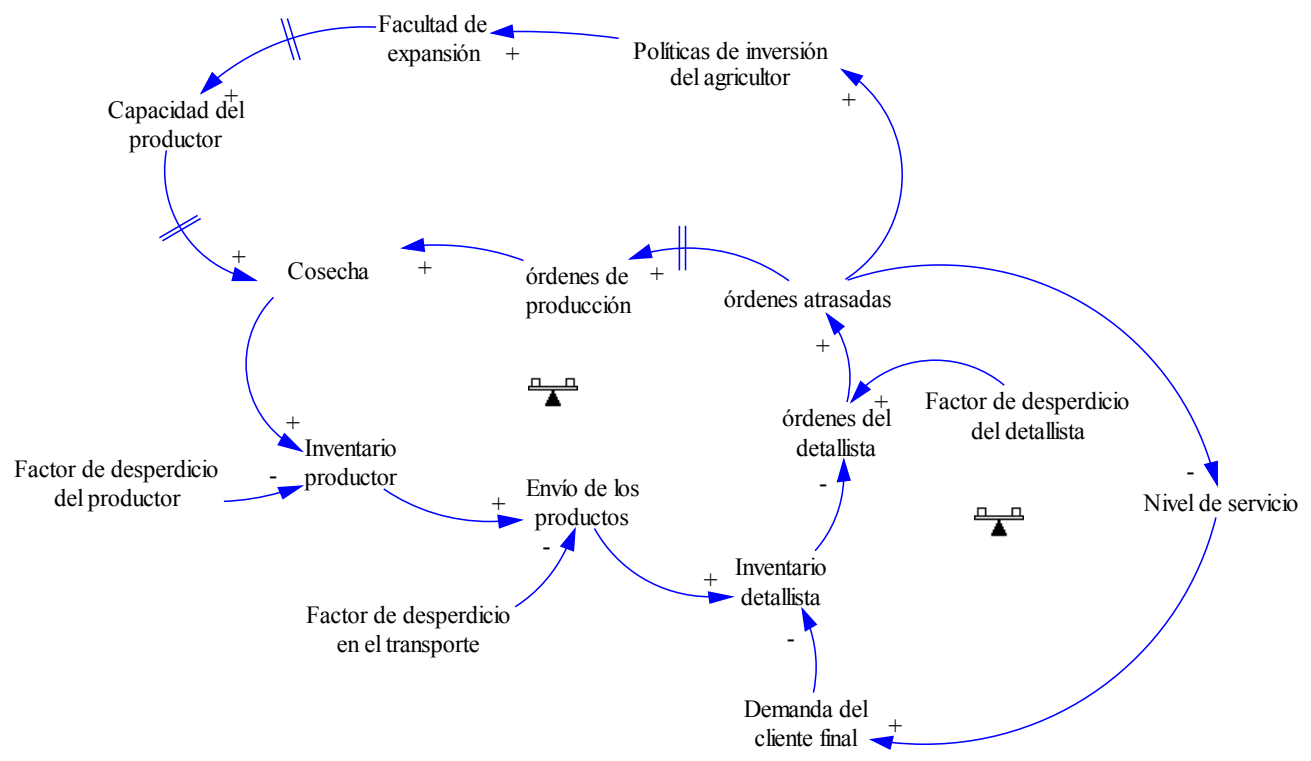


El segundo paso que plantea la metodología de dinámica de sistemas es crear un diagrama de Forrester (a partir del diagrama causal), el cual se constituye en la herramienta base para la simulación del estudio. Resumiendo, el modelo a ser descrito comprende los siguientes elementos y supuestos:

- Una cadena de suministros de dos eslabones, un detallista y un productor que atienden la demanda diaria del consumidor final

- La demanda del producto se considera con patrón perpetuo o uniforme

- Se distribuye un único producto

- Los tiempos de entrega entre el productory el cliente son variables entre 1 y 3 días

- La atención al cliente final es inmediata en el caso de contar con inventario

- Se consideran factores de desperdicio en la cosecha, el almacén del detallista y productor y en el transporte desde el productor a las instalaciones del detallista

- Se simula un periodo de 720 días equivalentes a dos años.

El diagrama Forrester completo para la cadena de suministro de la Papaya se muestra en la Figura 3. En la zona 1 se puede observar como el agricul- tor inicialmente tiene unas hectáreas sin sembrar y el proceso de cultivo de estas tiene una demora implícita debido a que las semillas de la Papaya no se cultivan directamente sobre el terreno, sino que existe un tiempo de siembra de estas en un vivero. Una vez la hectárea es plantada, debe esperar un lapso de tiempo para poder cosechar los frutos. Se debe tener en cuenta que una vez se ha recolectado la Papaya de una determinada hectárea, esta vuelve a estar disponible para la siembra.

En la demarcación 2 se puede observar como el productor abastece un producto el cual es un flujo de entrada para su inventario, cabe aclarar que esta producción dependerá de la capacidad inicial que el productor tenga, que en este estudio se ve representado por el número de hectáreas que el agricultor va adquiriendo y cosechando a través del tiempo. Además una vez se comience a recolectar la cosecha, existirá un factor de desperdicio asociado a pérdidas de producto en esta etapa.Un punto clave del estudio, es el hecho de que el producto debe estar entre 6 y 8 días en almacenamiento antes de ser enviado al detallista, debido a que la Papaya es una fruta climatérica que alcanza su punto de maduración óptimo, días después de ser retirada de la plantación.

Figura 3. Diagrama Forrester de la cadena de suministro de la Papaya

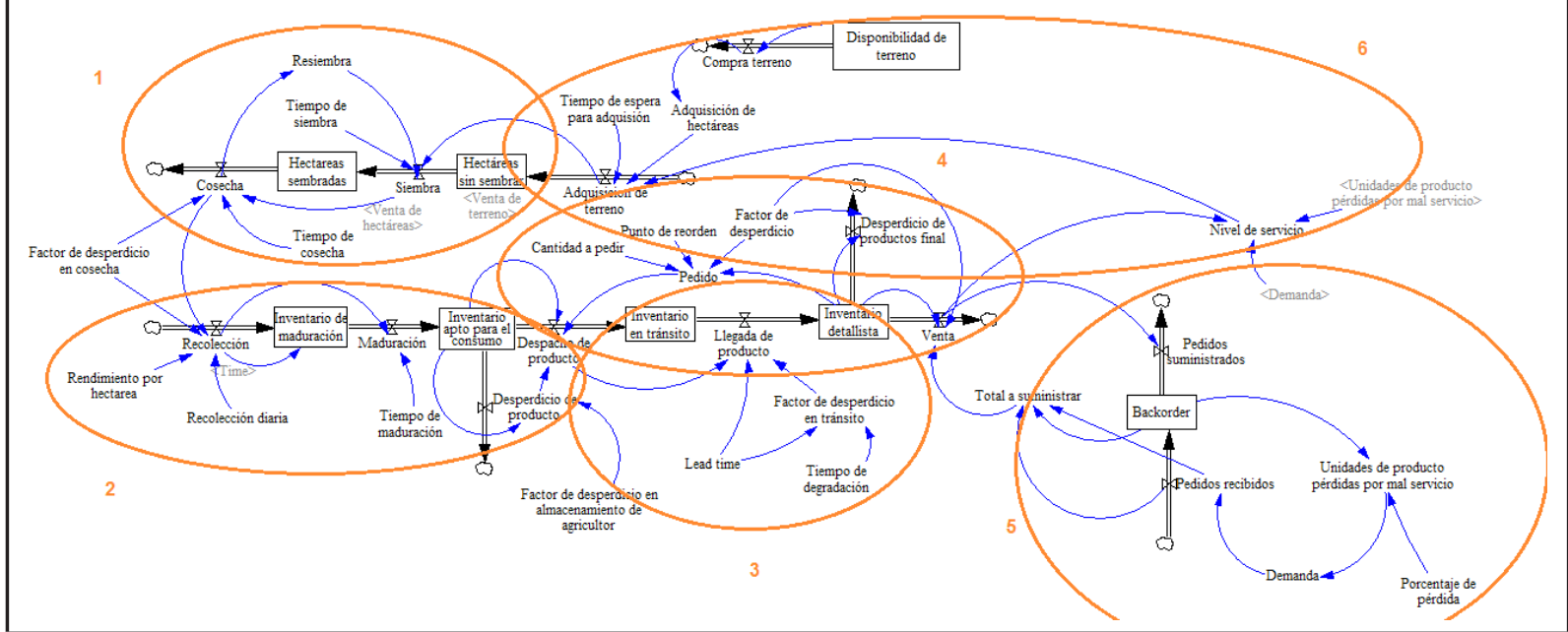


Debido a que la Papaya que es enviada por el productor no llega inmediatamente a su destino, se debe manejar un inventario en tránsito, cuya salida representará las unidades que llegan a suplir el inventario del detallista. Gracias al factor de desperdicio en el transporte, el cual se ve afectado por el tiempo que transcurre entre cada eslabón, las cantidades recibidas son menores a las que envió el productor (Ver zona 3).

En el círculo 4 se puede observar como el detallista responde a la demanda del cliente, sin embargo una parte de la Papaya que tiene almacenada en inventario se degrada y se pierde, por lo que no puede ser utilizada para la venta. Además el minorista realiza sus pedidos cada vez que el inventario efectivo esté por debajo del punto de reorden, y emitirá un pedido buscando cubrir la demanda promedio en el tiempo de reposición y a su vez, el factor de desperdicio inherente a un producto perecedero como lo es la Papaya.

Debido a que existe una gran posibilidad de incumplir con la demanda del cliente, por la degradación de los productos a través de toda la cadena de suministro, algunas órdenes son retrasadas (suponiendo que el cliente está dispuesto a esperar), lo que constituye el llamado Backorder. Sin embargo, si un pedido se demora demasiado tiempo en ser entregado al cliente, este no volverá a realizar órdenes de compra al detallista, debido a la mala imagen y bajo nivel de servicio que se le ha prestado (Ver zona 5).

El indicador que medirá la eficiencia de la cadena de suministro será el nivel de servicio, que representa la probabilidad esperada de no llegar a una situación de falta de existencias. La Flexibilidad de volumen en el modelo se ve representado por la adquisición de hectáreas. La política actual que utilizará el modelo es adquirir un cierto número de hectáreas sin sembrar cuando el nivel de servicio sea menor a un determinado porcentaje. Se debe aclarar que como la capacidad es una decisión estratégica y no se implementa inmediatamente, se tendrá el supuesto de que la decisión de compra o alquiler y la adecuación del terreno tendrán una demora implícita (Ver demarcación 6).

\section{RESULTADOS}

En el Gráfico 2 se puede observar que en el primer año simulado los pedidos suministrados, representados por la línea roja, son mucho menores al total de unidades que se debían suministrar, lo que significa que existen órdenes atrasadas y por lo tanto se está incumpliendo con los requisitos del cliente. Sin embargo, al principio del segundo año la política de aumento de capacidad implementada comienza a tener impacto sobre el desempeño de la cadena debido a que el detallista logra nivelar su producción con la demanda que presenta el cliente.

En el Gráfico 3 se puede notar como en el primer año, el costo de penalización de detallista es mayor que el costo de inventario del agricultor debido a que inicialmente el productor no tiene la suficiente capacidad para responder a las necesidades de los clientes. A partir del segundo periodo, el costo de inventario comienza a predominar ya que la política de incremento de flexibilidad de volumen ha provocado que el agricultor acumule una mayor cantidad de Papaya en su inventario, buscando reducir el costo por penalización generado por el incumplimiento a los clientes.

\section{Gráfico 2. Total a suministrar vs Pedidos suministrados}

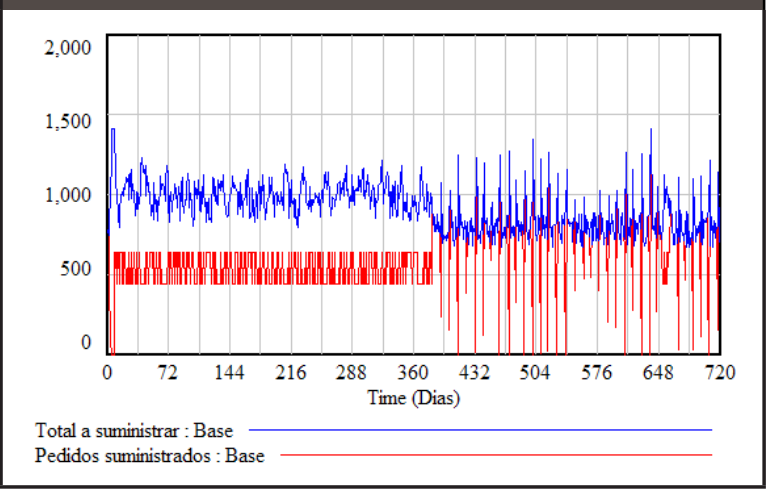


Gráfico 3. Costo de inventario agricultor vs costo penalización detallista

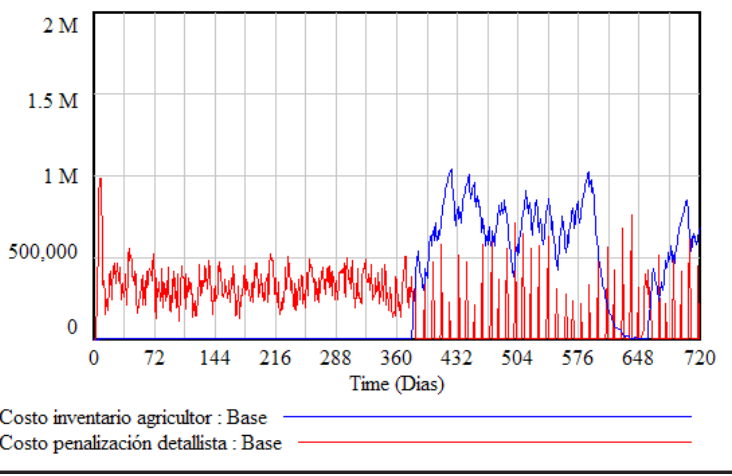

El nivel de servicio del detallista al inicio de la simulación se mantiene por debajo del nivel deseado (45,35\% en promedio) por lo que el productor constantemente está adquiriendo capacidad para mejorar el nivel de servicio de la cadena; sin embargo, esta decisión solo se viene a ver reflejada al principio del segundo año debido a la demora implícita que tiene un proceso de aumento de capacidad. En esta última etapa, el detallista comienza a cumplir con las unidades que el cliente ha demandado y con los pedidos pendientes, si es que se tienen; lo que se ve representado en un nivel de servicio cercano al 100\%. El nivel de servicio promedio obtenido durante la simulación es de $64,17 \%$ un indicador que aunque no es cercano al objetivo de un nivel de servicio del $100 \%$ que buscan todas las cadenas de suministro, se convierte en un indicador aceptable para este tipo de sistemas complejos.

Por último, como se puede observar en el Gráfico 4, los factores de desperdicio en el sistema generan que en el primer año de simulación no se logre satisfacer las necesidades del cliente y por ende las unidades pendientes a entregar al cliente son superiores al producto almacenado en los inventarios del productor y detallista; este incumplimiento en la demanda genera que se comiencen a emitir pedidos constantemente por parte del minorista $y$ se cree una demanda sobreevaluada, a partir de la cual el agricultor adquiere hectáreas para producir y enviar una mayor cantidad de Papaya al detallista; sin embargo, esta sobrevaloración de las demanda provoca que en el segundo año la capacidad adquirida por el productor sea mucho mayor a la demanda del mercado, hecho reflejado en los altos niveles de inventario que alcanzan tanto el agricultor como el detallista. A este desajuste entre la demanda real de los consumidores y la generada por los actores de la cadena de suministro, se le conoce como Efecto Látigo. Por lo tanto, se puede evidenciar la aparición de un efecto látigo por la consideración de factores de desperdicio que generan pedidos más frecuentes $\mathrm{y}$ de mayor volumen al proveedor y los eslabones anteriores a él.

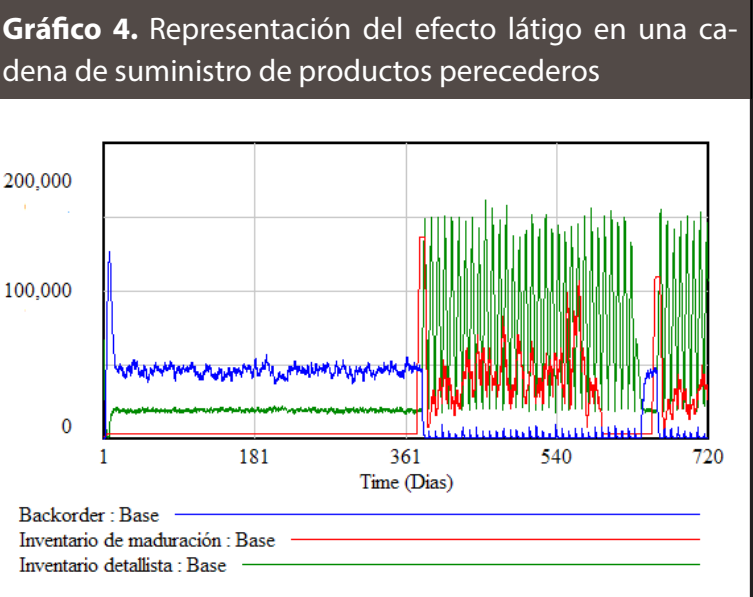

\subsection{Análisisis de sensibilidad}

Luego de simular el modelo base, se realizó un análisis de sensibilidad de algunos parámetros del modelo caso de estudio buscando encontrar la influencia que tiene cada uno de estos sobre el comportamiento y desempeño de la cadena de suministro de Papaya.

En el Gráfico 5 se evidencia el inventario apto para el consumo que tiene el agricultor, producto de la variación consecutiva en un $20 \%$ del factor de desperdicio inicial en el almacenamiento del productor (8\%). Se puede evidenciar que a partir del segundo año, que es el momento en que la política de flexibilidad de volumen comienza a afectar al suministro de Papaya del productor, el nivel de inventario es más alto cuando se tiene un menor factor 
de desperdicio, debido a que la degradación dentro del almacenamiento es menor. Además, a medida de que el desperdicio en inventario del productor es mayor, la entrega de Papayas al detallista se reduce, por lo que los pedidos pendientes en promedio se incrementan hasta en un $99 \%$ comparado con el caso inicial (Ver Tabla 1).

\begin{tabular}{|c|c|c|c|}
\hline $\begin{array}{c}\text { Factor de } \\
\text { desperdicio }\end{array}$ & Máximo & Promedio & $\begin{array}{l}\text { Variación } \\
\text { respecto al } \\
\text { caso inicial }\end{array}$ \\
\hline $48 \%$ & 1424 & 576 & $99 \%$ \\
\hline $28 \%$ & 1416 & 440 & $52 \%$ \\
\hline $8 \%$ & 1408 & 290 & $0 \%$ \\
\hline
\end{tabular}

En el Gráfico 6 se muestra las hectáreas cultivadas a través del tiempo, resultado de una variación del $20 \%$ en el factor de desperdicio inicial (5\%) en el almacenamiento del detallista. Se puede notar como a partir del día 150 (representado por la línea amarilla), las decisiones de incremento de capacidad se comienzan a hacer notorias: a medida que el factor de desperdicio toma niveles más altos, se pierde una mayor cantidad de Papaya en el almacén del detallista, lo que provoca que este realice pedidos constantes y grandes de Papaya, debido que no alcanza a suplir la demanda de su cliente con lo poco que le queda en inventario. El agricultor al percibir esa alza en los pedidos del detallista y el bajo nivel de servicio que le está ofreciendo a su cliente, se ve obligado a adquirir hectáreas para poder suministrar un mayor número de Papayas a la cadena. En pocas palabras, entre mayor sea el factor de desperdicio asociado al almacenamiento del detallista, mayor serán las hectáreas cultivadas por el agricultor. Sin embargo, como el productor tiene un límite en la disponibilidad de terreno para cultivar, llega el momento en que a pesar de que su desperdicio sea muy grande, este no podrá sembrar más hectáreas. Al ser mayor el factor de desperdicio en el almacenamiento del detallista, la planificación de la demanda de este se ve afectada debido a que ahora debe pedir una mayor cantidad de Papaya para cubrir la misma demanda porque se pierde mucho producto en el recorrido por la cadena, provocando que se pierdan, en una mayor proporción, unidades demandas por algunos clientes (hasta en un $122 \%$ más que el caso inicial, como se puede ver en la Tabla 2) debido al constante incumplimiento de los pedidos por parte del minorista.

Gráfico 5. Comportamiento del inventario del agricultor frente a una variación del factor de desperdicio en el almacén del productor

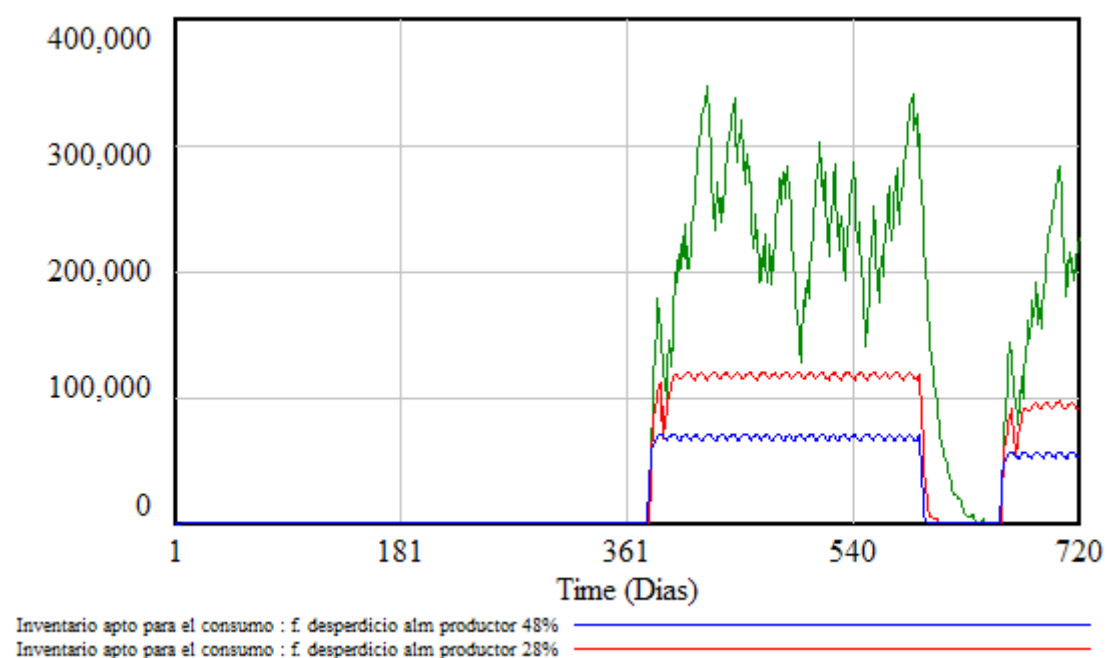

Inventario apto para el consumo : 4 desperdicio alm productor 4896

Inventario apto para el consumo : f desperdicio alm productor $28 \%$
Inventario apto para el consumo : f desperdicio alm productor $8 \%$ 
Por ejemplo, mientras que en el caso inicial en el que se tenía un factor de deterioro de producto en el almacén del $5 \%$, se perdían en promedio una demanda de 152 Papayas, el escenario en que este valor se incrementa a un $45 \%$ ocasiona la pérdida de demanda de 337 Papayas.

TABLA 2. INDICADORES DEDEMANDA PÉRDIDA FRENTE A UNA VARIACIÓN DEL FACTOR DE DESPERDICIO DEL DETALLISTA

\begin{tabular}{c|c|c|c}
$\begin{array}{c}\text { Factor de } \\
\text { desperdicio }\end{array}$ & $\begin{array}{c}\text { Máximo } \\
\text { (Papayas) }\end{array}$ & $\begin{array}{c}\text { Promedio } \\
\text { (Papayas) }\end{array}$ & $\begin{array}{c}\text { Variación } \\
\text { respecto al } \\
\text { caso inicial }\end{array}$ \\
\hline $45 \%$ & 784 & 337 & $122 \%$ \\
\hline $25 \%$ & 771 & 244 & $60 \%$ \\
\hline $5 \%$ & 739 & 152 & $0 \%$ \\
\hline
\end{tabular}

Por último, se realizó una variación simultánea del $10 \%$ y $20 \%$ a cada factor de degradación de la cadena actual de suministro de la Papaya. La Tabla 3 exhibe como al ser mayor el factor de desperdicio en la cadena de suministro, se pierde una mayor cantidad de producto en la cadena, lo que ocasiona que el detallista incumpla sus pedidos en una mayor proporción y esto afecta al costo de penalización asociado a los faltantes ya que en el peor de los casos, los costos asociados al incumplimiento de los pedidos se incrementan hasta en un $113 \%$, lo que genera que la rentabilidad de las compañías disminuya.

TABLA 3. COSTO DE PENALIZACIÓN DE DETALLISTA FRENTE A UNA VARIACIÓN DEL DESPERDICIO GLOBAL DE LA CADENA DE SUMINISTRO

\begin{tabular}{c|c|c|c|}
$\begin{array}{c}\text { Variación } \\
\text { desperdicio }\end{array}$ & Máximo & Promedio & $\begin{array}{c}\text { Variación } \\
\text { respecto al } \\
\text { caso inicial }\end{array}$ \\
\hline $20 \%$ & $\$ 1.033 .403$ & $\$ 432.250$ & $113 \%$ \\
\hline $10 \%$ & $\$ 1.012 .669$ & $\$ 335.073$ & $65 \%$ \\
\hline Caso Base & $\$ 985.414$ & $\$ 202.692$ & $0 \%$ \\
\hline
\end{tabular}

En el Gráfico 7 se evidencia el impacto sobre el nivel de servicio, que tiene una variación incremental del 5\% en cada uno de los factores de desperdicio de la cadena. En este gráfico se puede observar como un incremento en el desperdicio de Papaya en la cadena de suministro ocasiona que el nivel de servicio sea menor respecto al caso inicial; aspecto que hace necesario llevar un estricto control de este tipo de cadenas para reducir los factores que generan la degradación de la Papaya y de esa forma mejorar el nivel de servicio de todo el sistema.

Gráfico 6. Hectáreas cultivadas frente a una variación del factor de desperdicio en el almacén del detallista

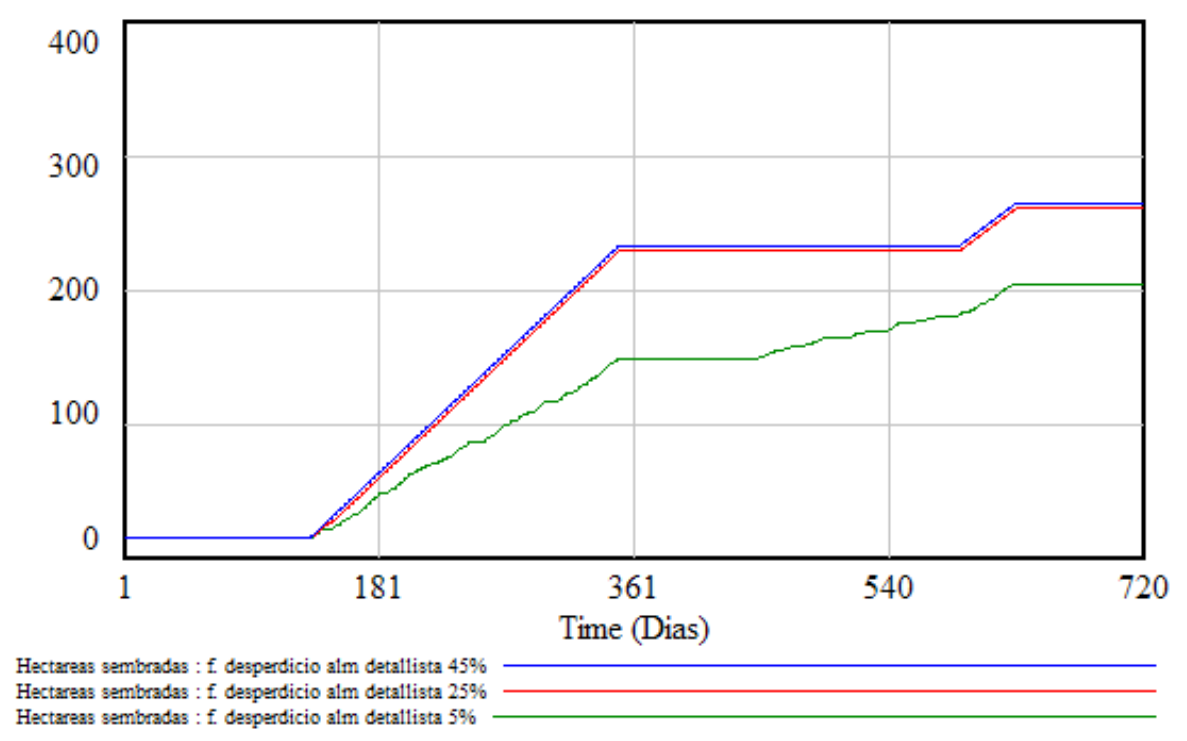


Gráfico 7. Comportamiento del nivel de servicio frente a una variación del desperdicio generado en la cadena de suministro

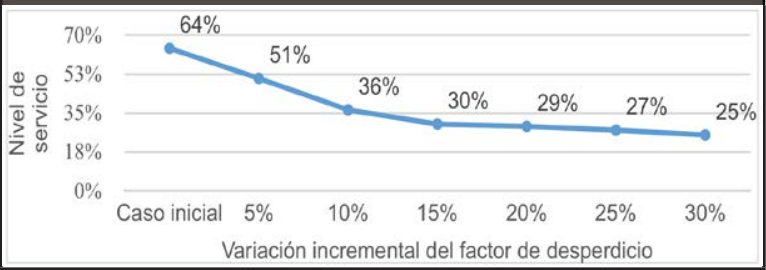

El Gráfico 8 exhibe el comportamiento del nivel de servicio respecto al costo que conlleva obtener dicho nivel de servicio. Primero se puede notar que un nivel de servicio del $100 \%$ es una meta utópica debido a que en la realidad es prácticamente imposible por la aleatoriedad presente en la demanda de los clientes. Además se puede observar que existe un momento en que una respectiva inversión puede generar una mejora significativa en el nivel de servicio, evidenciado en el hecho de que el cambio en la variable costo es menor que el cambio en el nivel de servicio. En el momento en que el nivel de servicio varíe en una menor proporción que el costo, se debe evaluar qué porcentaje de ingresos adicionales se obtiene debido a la mejora en la atención de las necesidades del cliente, para poder comparar si la inversión realizada en mejorar la satisfacción del cliente final se ve compensada con los mayores ingresos por venta del producto.

Gráfico 8. Comportamiento del nivel de servicio frente al costo de inversión en capacidad adicional

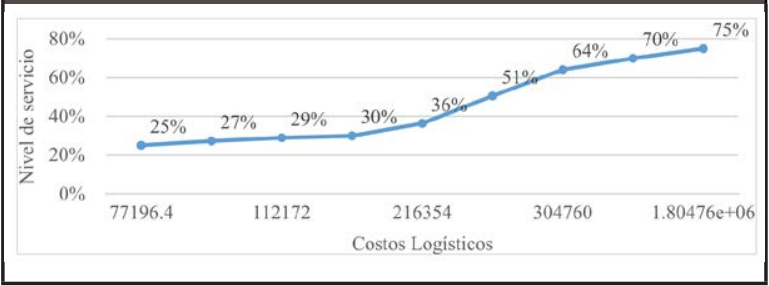

\subsection{Análisis por escenarios}

Por último, se plantearon dos escenarios diferentes al inicialmente propuesto para ver el impacto que tiene la consideración de algunos supuestos dentro del modelo.

El primer escenario consiste en realizar un análisis comparativo entre dos cadenas de suminis- tro: una que maneja productos perecederos y otra que no; todo con el objetivo de identificar el impacto que tiene la consideración del desperdicio en términos de costo y nivel de servicio.

En este escenario, la necesidad de incrementar capacidad es más frecuente en una cadena de suministro que involucra el manejo de productos perecederos, debido a que como el inventario que tiene el detallista es menor, se genera un mayor incumplimiento en las unidades que demanda el cliente y por ende el nivel de servicio alcanza niveles críticos, lo que hace que el costo de penalización del detallista sea mayor (aproximadamente en un 773\%) en una cadena de suministro que distribuye productos perecederos (Ver Tabla 4).

TABLA 4. COSTO DE PENALIZACIÓN DE DETALLISTA EN UNA CADENA DE SUMINISTRO CONY SIN DESPERDICIO

\begin{tabular}{c|c|c} 
Escenario & Promedio & Variación \\
\hline CS sin desperdicio & $\$ 23.228$ & $0 \%$ \\
\hline CS con desperdicio & $\$ 202.692$ & $773 \%$ \\
\hline
\end{tabular}

El proveedor a evidenciar los bajos niveles de servicio que está teniendo el detallista, comienza a implementar políticas de incremento de flexibilidad de volumen, específicamente de aumento de capacidad, que en este caso consiste en cultivar un mayor número de hectáreas de Papaya, lo que ocasiona que las hectáreas cultivadas en una cadena de suministro de productos perecederos sean mayores comparación con una cadena de suministro que no maneja productos de corto ciclo de vida. Como consecuencia de este hecho, la implementación constante de la política de flexibilidad de volumen en la cadena de suministro de productos perecederos genera un mayor costo asociado al aumento de capacidad comparado con un sistema logístico que maneja productos no perecederos.

Comparando los niveles de servicio de una cadena de suministro que maneja un producto perecedero como la Papaya, con una red que distribuye un producto que no tiene desperdicio asociado, se puede observar como el nivel de servicio es menor en el primer caso $(64,17 \%$ vs $94,71 \%$, ver Tabla 5),

Revista EIA Rev.EIA.Esc.Ing.Antioq / Universidad EIA 
debido a que en el caso de la cadena de suministro de productos perecederos, el detallista a la hora de realizar los pedidos no solo debe tener en cuenta la demanda del cliente, sino también el factor de desperdicio generado en todo el sistema; lo que ocasiona que si este no planifica bien su demanda, puede presentar escenarios de venta pérdida o backorder que terminan afectando el nivel de servicio de toda la cadena.

Por último, como se puede observar en la Tabla 5 , una cadena de suministro de productos perecederos involucra costos logísticos más altos (aproximadamente en un 912\%): generados por pedidos más frecuentes, costos de almacenamiento más altos y mayores penalizaciones por un bajo nivel de servicio, comparados con una red que distribuye un producto no perecedero. Además la variación de los costos a través del tiempo es más notaria en una cadena de suministro de un producto perecedero, hecho reflejado en el mayor valor que alcanza la desviación estándar para el sistema en el que se considera desperdicio.

En el segundo escenario se compararon dos cadenas de suministro de productos perecederos: la primera, en la cual se aplica una política de flexibilidad de volumen, vista desde el punto de vista de un aumento de capacidad de producción del proveedor; y la segunda, un sistema en el cual no existe tal política de flexibilidad. Esta comparación se hace con el objetivo de establecer el impacto que tiene una política de flexibilidad de volumen sobre algunos indicadores de gestión de cadena de suministro como lo son el nivel de servicio y los costos logísticos. Los resultados más relevantes se presentan a continuación.

En la Tabla 6, se puede notar como las unidades pendientes a entregar al cliente son mayores en el caso en que no se utiliza la política de flexibilidad de volumen, debido a que el proveedor nunca aumenta su capacidad de producción para generar mayor fluidez de producto hacia el detallista, lo que hace que este le siga incumpliendo a su cliente y el nivel de servicio alcance niveles críticos en comparación con una cadena de suministro en la cual se aplica la política de flexibilidad de volumen. En la Tabla 6, se puede notar como el nivel de servicio promedio de una cadena de suministro de productos perecederos que no utiliza una política de flexibilidad de volumen, es mucho menor comparado con el que caso en la que si se utiliza esta estrategia ( $49.3 \%$ vs $64.2 \%$ ).

TABLA 6. BACKORDER PROMEDIO Y NIVEL DE SERVICIO PROMEDIO EN UNA CADENA DE SUMINISTRO CON Y SIN POLÍTICA DE FLEXIBILIDAD DE VOLUMEN

\begin{tabular}{c|c|c|} 
Escenario & $\begin{array}{c}\text { Backorder } \\
\text { promedio }\end{array}$ & $\begin{array}{c}\text { Nivel de servicio } \\
\text { promedio }\end{array}$ \\
\hline $\begin{array}{c}\text { CS sin política de } \\
\text { flexibilidad de } \\
\text { volumen }\end{array}$ & 436,7 & $49,3 \%$ \\
\hline $\begin{array}{c}\text { CS con política } \\
\text { de flexibilidad de } \\
\text { volumen }\end{array}$ & 289,6 & $64,2 \%$ \\
\hline
\end{tabular}

En el caso en que una cadena de suministro maneja una política de aumento de capacidad, el proveedor incurre en una menor penalización, comparado con el caso en que no se usa la política de flexibilidad (Ver Tabla 7), debido a que sus entregas al detallista se ajustan en una mayor proporción al pedido que este realiza. Esto a su vez ocasiona que el detallista, en el caso en que no se usa una estrategia de flexibilidad, tenga un mayor costo asociado al mal servicio prestado al cliente (ver Tabla 7), ya que en ningún momento el proveedor aumenta sus pedidos debido a la capacidad fija y limitada que este posee.

TABLA 5. COSTOS LOGÍ́STICOS EN UNA CADENA DE SUMINISTRO CON Y SIN DESPERDICIO

\begin{tabular}{c|c|c|c|c|} 
Escenario & Promedio & Variación & Desviación estándar & Nivel de servicio promedio \\
\hline CS sin desperdicio & $\$ 51.460$ & $0 \%$ & 103.742 & $94.71 \%$ \\
\hline CS con desperdicio & $\$ 520.749$ & $912 \%$ & 262.630 & $64.17 \%$ \\
\hline
\end{tabular}


TABLA 7. IMPACTO DE UNA POLÍTICA DE FLEXIBILIDAD DE VOLUMEN SOBRE EL COSTO PROMEDIO DE PENALIZACIÓN DEL PRODUCTOR Y AL DETALLISTA

\begin{tabular}{|c|c|c|} 
Escenario & $\begin{array}{c}\text { Costo promedio } \\
\text { penalización } \\
\text { productor }\end{array}$ & $\begin{array}{c}\text { Costo promedio } \\
\text { penalización } \\
\text { detallista }\end{array}$ \\
\hline $\begin{array}{c}\text { CS sin política } \\
\text { de flexibilidad } \\
\text { de volumen }\end{array}$ & 305.655 & 18.090 \\
\hline $\begin{array}{c}\text { CS con política } \\
\text { de flexibilidad } \\
\text { de volumen }\end{array}$ & 202.692 & 13.296 \\
\hline
\end{tabular}

Un aspecto que se debe resaltar es como con la política de aumento de capacidad, se incrementan los niveles de inventario del productor, lo que ocasiona que el costo de mantenimiento de este sea mucho mayor en comparación con un proveedor que no utiliza una estrategia de flexibilidad de volumen.

En conclusión se puede decir que la política de flexibilidad de volumen mejora notablemente indicadores como el nivel de servicio y los costos asociados a las penalizaciones del productor y detallista. Sin embargo, utilizar esta estrategia implica asumir un mayor costo de almacenamiento de inventario y un costo adicional de aumento de capacidad, que en la simulación está representado por el valor que tiene la compra o alquiler de cada hectárea.

\section{CONCLUSIONES}

El análisis de la flexibilidad de volumen en cadenas de suministro de productos perecederos, realizada en esta investigación, busca ayudar a unificar y sistematizar una serie de conceptos relativos a la gestión de este tipo de cadenas tales como la planificación de la demanda, la política de pedido, los niveles de inventarios, etc.

Se ha encontrado que las decisiones de flexibilidad deben considerar no sólo el comportamiento de la demanda sino el tipo de producto que se distribuye, las características perecederas de un producto influyen sobre los pedidos y la capacidad necesaria para atenderlos. Se concibe la aparición de un efecto látigo por la consideración de factores de desperdicio que generan pedidos más frecuentes y de mayor volumen al proveedor y los eslabones anteriores a él. Otro aspecto que influencia la gestión de inventarios del detallista es el factor de desperdicio asociado al proceso de distribución. En conjunto se resalta la influencia negativa sobre el control del inventario ya que dichos factores de desperdicio distorsionan la información de demanda del cliente final para todas las firmas integradas en una cadena de suministro.

Se puede plantear que existe un retraso entre la decisión gerencial de incremento de la capacidad del proveedor y el efecto positivo que pueda tener en los niveles de servicio al cliente final. Para el caso puntual del estudio se encontró que el beneficio se empieza a manifestar después de 1 año de operación.

La Dinámica de Sistemas se considera relevante como insumo gerencial para analizar los impactos que tienen las decisiones de política, como lo fue en este caso el incremento de capacidad frente a un determinado nivel de pedidos pendientes. Es posible evaluar otras políticas así como el flujo de información entre los participantes de la cadena, con lo cual se establece un rango amplio de estudio sobre la flexibilidad aún sin explorar, y que a su vez es soportado por la misma revisión bibliográfica realizada en este trabajo, que señala la falta de estudios en este tema.

La gestión de cadenas de suministro de productos perecederos como la papaya es mucho más compleja que la de un producto no perecedero debido principalmente a la distorsión en la demanda que causa el deterioro del producto a lo largo de la cadena.

Para cualquier cadena de suministro llegar a un nivel de servicio del $100 \%$ es una meta utópica debido a que en la realidad es prácticamente imposible por la aleatoriedad presente en la demanda de los clientes. Además se puede observar que existe un momento en que una respectiva inversión puede generar una mejora significativa en el nivel de servicio, evidenciado en el hecho de que el cambio en la variable costo es menor que el cambio en el nivel de servicio. En el momento en que el nivel de ser- 
vicio varíe en una menor proporción que el costo, se debe evaluar qué porcentaje de ingresos adicionales se obtiene debido a la mejora en la atención de las necesidades del cliente, para poder comparar si la inversión realizada en mejorar la satisfacción del cliente final se ve compensada con los mayores ingresos por venta del producto.

Una cadena de suministro de productos perecederos involucra costos logísticos más altos: generados por pedidos más frecuentes, costos de almacenamiento más altos y mayores penalizaciones por un bajo nivel de servicio, comparados con una red que distribuye un producto no perecedero.

La política de flexibilidad de volumen mejora notablemente indicadores como el nivel de servicio y los costos asociados a las penalizaciones del productor y detallista. Sin embargo, utilizar esta estrategia implica asumir un mayor costo de almacenamiento de inventario y un costo adicional de aumento de capacidad, que en la simulación está representado por el valor que tiene la compra o alquiler de cada hectárea.

\section{REFERENCIAS}

Bhunia, a. K., \& Maiti, M. (1999). An inventory model of deteriorating items with lot-size dependent replenishment cost and a linear trend in demand. Applied Mathematical Modelling, 23(4), 301-308. http:// doi.org/10.1016/S0307-904X(98)10089-6

Blome, C., Schoenherr, T., \& Eckstein, D. (2014). The impact of knowledge transfer and complexity on supply chain flexibility: A knowledge-based view. International Journal of Production Economics, 147, 307316. http://doi.org/10.1016/j.ijpe.2013.02.028

Braunscheidel, M. J., \& Suresh, N. C. (2009). The organizational antecedents of a firm's supply chain agility for risk mitigation and response. Journal of $O p$ erations Management, 27(2), 119-140. http://doi. org/10.1016/j.jom.2008.09.006

Castellanos, O., Fúquene, A., Fonseca, S., Ramírez, D., Giraldo, E., \& Valencia, M. (2011). ESTUDIO DE LA CADENA PRODUCTIVA DE LA PAPAYA EN LA REGIÓN DEL NORTE DEL VALLE-BRUT.

Croom, S., Romano, P., \& Giannakis, M. (2000). Supply chain management: an analytical framework for critical literature review. European Journal of Purchasing \& Supply Management, 6(1), 67-83. http:// doi.org/10.1016/S0969-7012(99)00030-1

Disney, S. M., \& Towill, D. R. (2003). The effect of vendor managed inventory (VMI) dynamics on the Bullwhip Effect in supply chains. International Journal of Production Economics, 85(2), 199-215. http:// doi.org/10.1016/S0925-5273(03)00110-5

Higuchi, T., \& Troutt, M. D. (2004). Dynamic simulation of the supply chain for a short life cycle productLessons from the Tamagotchi case. Computers \& Operations Research, 31(7), 1097-1114. http://doi. org/10.1016/S0305-0548(03)00067-4

Jack, E. P., \& Raturi, A. (2002). Sources of volume flexibility and their impact on performance. Journal of $\mathrm{Op}$ erations Management, 20(5), 519-548. http://doi. org/10.1016/S0272-6963(01)00079-1

Lee, H. L., Padmanabhan, V., \& Whang, S. (1997). The bullwhip effect in supply chains. MIT Sloan Management Review, 38(3), 93-102.

Li, R., Hongjie, L., \& Mawhinney, J. (2010). A Review on Deteriorating Inventory Study. Journal of Service Science and Management, 03(01), 117-129.

Minegishi, S., \& Thiel, D. (2000). System dynamics modeling and simulation of a particular food supply chain. Simulation Practice and Theory, 8(5), 321-339. http://doi.org/10.1016/S0928-4869(00)00026-4

Nita.H. Shah. (1993). Probabilistic time-scheduling model for an exponentially decaying inventory when delays in payments are permissible. International Journal of Production Economics, 32(1), 77-82.

Padmanabhan, G., \& Vrat, P. (1995). EOQ models for perishable items under stock dependent selling rate. European Journal of Operational Research, 86(2), 281-292.

Paredes, A. M., \& Salazar, A. F. (2014). Visión sistémica del análisis de la flexibilidad en cadenas de suministro de productos perecederos. Sistemas \& Telemàtica, 63-86.

Sicilia, J., González, M., Febles, J., \& Alcaide, D. (2014). An inventory model for deteriorating items with shortages and time-varying demand. International Journal of Production Economics, (2003), 1-8.

Stevenson, M., \& Spring, M. (2007). Flexibility from a supply chain Flexibility perspective: definition and review. International Journal of Operations \& Pro- 
duction Management, 27(7), 685-713. http://doi. org/10.1108/01443570710756956

Vickery, S., Calantone, R., \& Dröge, C. (1999). Supply Chain Flexibility: An empirical study. Journal of Supply Chain Management, 35(3), 16-24.

Zhang, Q., Vonderembse, M. A., \& Lim, J.-S. (2003). Manufacturing flexibility: defining and analyzing relationships among competence, capability, and customer satisfaction. Journal of Operations Management, 21(2), 173-191. http://doi.org/10.1016/S02726963(02)00067-0

Paredes Rodríguez, A.M.; Salazar Ramos, A.F. (2017). Evaluación sistémica de una política de flexibilidad de volumen en una cadena de suministro distribuidora de papaya. Revista EIA, 14(27), enero-junio, pp. 43-62. [Online]. Disponible en: https://doi.org/10.24050/reia.v14i27.865

Revista EIA Rev.EIA.Esc.Ing.Antioq / Universidad EIA 


\section{ANEXOS}

Anexo 1. Cuadro resumen de revisión literaria

sobre el concepto de flexibilidad y sus aplicaciones

\begin{tabular}{|c|c|c|c|c|c|c|c|c|c|c|c|}
\hline $\begin{array}{l}\text { Método } \\
\text { utilizado }\end{array}$ & $\frac{\pi}{z}$ & 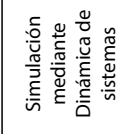 & 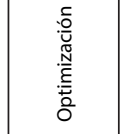 & $\frac{\pi}{z}$ & 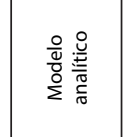 & $\frac{\pi}{z}$ & 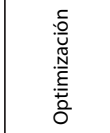 & $\frac{s}{z}$ & 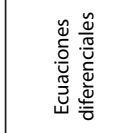 & $\Sigma$ & 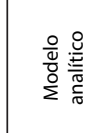 \\
\hline $\begin{array}{l}\text { Caso de } \\
\text { estudio }\end{array}$ & 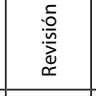 & $\begin{array}{l}\stackrel{8}{\overline{0}} \\
\stackrel{0}{\circ}\end{array}$ & 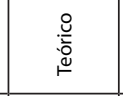 & 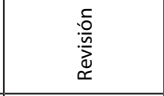 & 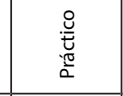 & 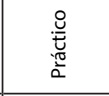 & : & 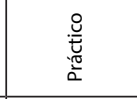 & : & 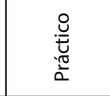 & 䓂 \\
\hline $\begin{array}{l}\text { Patrón de } \\
\text { demanda }\end{array}$ & $\frac{s}{z}$ & 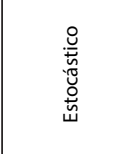 & 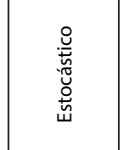 & $\frac{\pi}{2}$ & 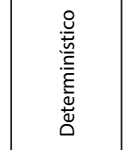 & $\begin{array}{l}\stackrel{8}{0} \\
\text { 莺 } \\
\text { 离 }\end{array}$ & 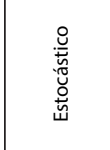 & 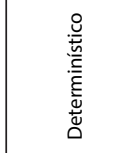 & 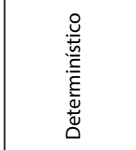 & $\mathbb{z}$ & 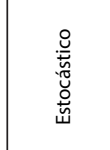 \\
\hline $\begin{array}{c}\text { Otras } \\
\text { caracteristicas }\end{array}$ & $\frac{\pi}{z}$ & $\frac{\pi}{z}$ & $\underline{z}$ & $\frac{\pi}{z}$ & 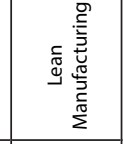 & $\frac{\pi}{z}$ & 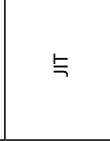 & $\frac{\pi}{z}$ & $\frac{\pi}{z}$ & 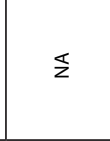 & $\triangleq$ \\
\hline Industria & $\underline{s}$ & 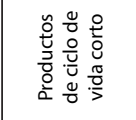 & $\underline{z}$ & $\frac{\pi}{z}$ & $\frac{\pi}{z}$ & $\frac{\pi}{z}$ & $\underline{z}$ & $\Sigma$ & 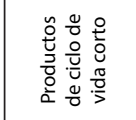 & $\frac{s}{z}$ & $\Sigma$ \\
\hline Respuesta & & $\times$ & & & & & & & $x$ & & \\
\hline Distribución & & & $x$ & & & & & & & & \\
\hline $\begin{array}{c}\text { Nuevo } \\
\text { producto }\end{array}$ & & & & $\times$ & & & & & $\times$ & & \\
\hline Volumen & $\times$ & $x$ & $\times$ & $\times$ & $x$ & $x$ & $\times$ & $\times$ & $\times$ & $\times$ & $\times$ \\
\hline Producto & & & $\times$ & $\times$ & $\times$ & & & $\times$ & & & \\
\hline Campo & $\frac{s}{z}$ & 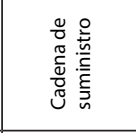 & 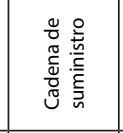 & $\frac{\pi}{z}$ & 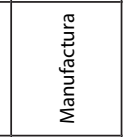 & 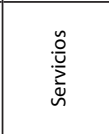 & 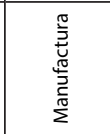 & 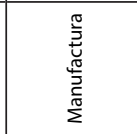 & 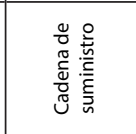 & 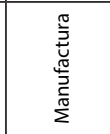 & 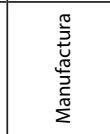 \\
\hline $\begin{array}{c}\text { Forma de } \\
\text { flexibilidad de } \\
\text { volumen }\end{array}$ & 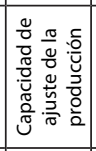 & 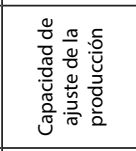 & 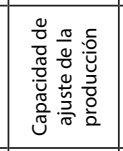 & 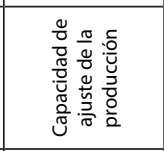 & 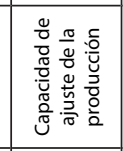 & 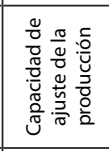 & 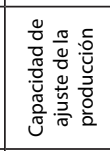 & 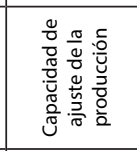 & 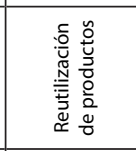 & 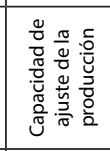 & 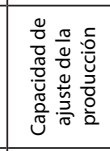 \\
\hline Año & ర్ & ò & $\overline{\grave{i}}$ & o. & $\stackrel{m}{i}$ & o̊̀ & ఃั & ì & $\sum_{i}^{m}$ & ఫ্ণ & $\overline{\bar{\alpha}}$ \\
\hline Revista & 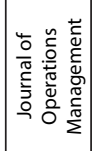 & 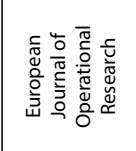 & 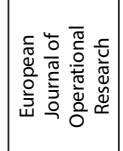 & 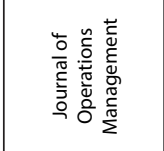 & 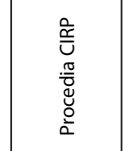 & 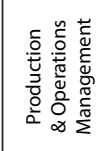 & 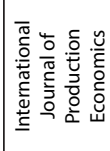 & 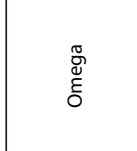 & 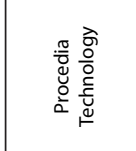 & 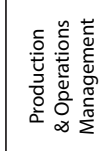 & 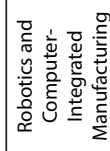 \\
\hline Autores & 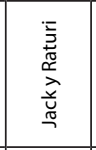 & 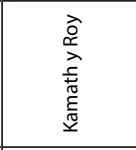 & $\stackrel{\square}{0}$ & 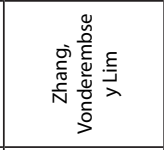 & 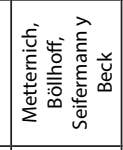 & 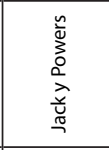 & 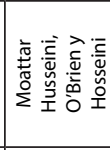 & 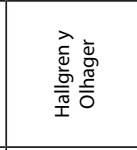 & $\begin{array}{l}\text { c } \\
\text { के } \\
\text { ज़ } \\
\text { ते } \\
\text { के } \\
\text { ज्ञ }\end{array}$ & 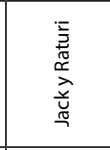 & 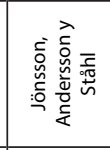 \\
\hline Nombre & 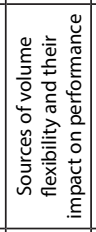 & 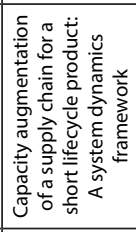 & 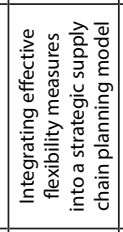 & 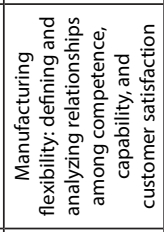 & 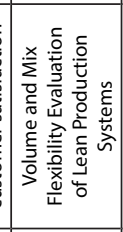 & 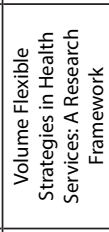 & 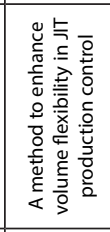 & 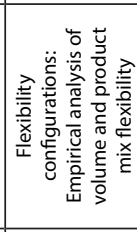 & 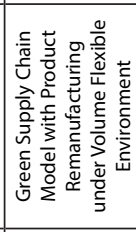 & 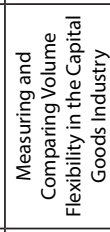 & 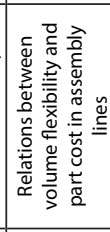 \\
\hline item & - & $\sim$ & m & $\sigma$ & in & ${ }^{\circ}$ & $n$ & $\infty$ & $a$ & 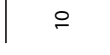 & $=$ \\
\hline
\end{tabular}




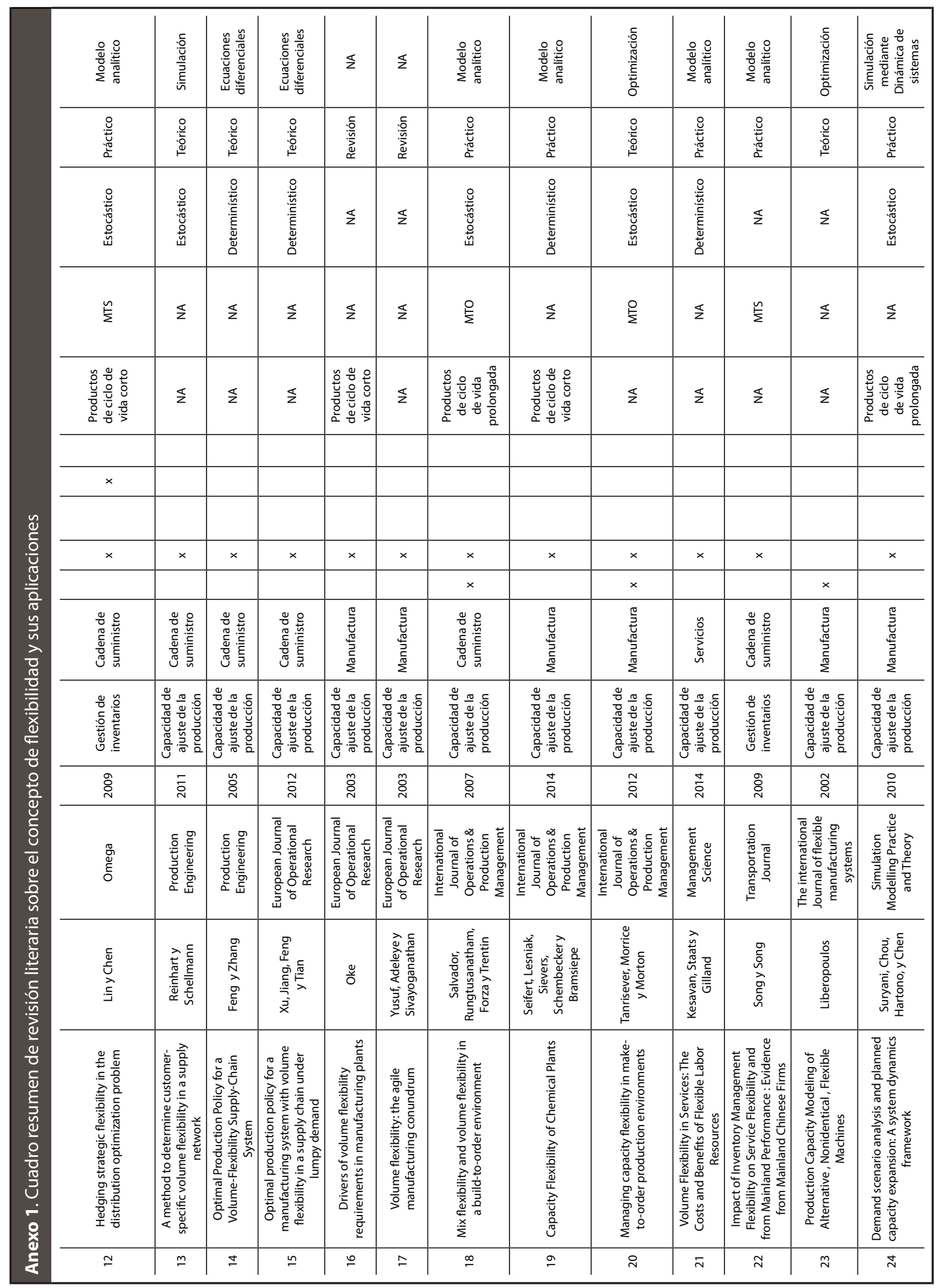




\begin{tabular}{|c|c|c|c|c|c|c|c|c|c|c|c|c|}
\hline 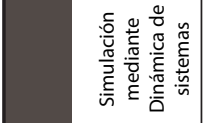 & $\underline{\Sigma}$ & $\underline{z}$ & 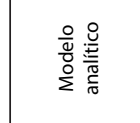 & $\underline{\Sigma}$ & 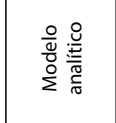 & 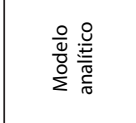 & 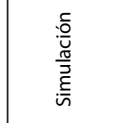 & 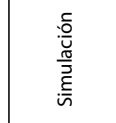 & 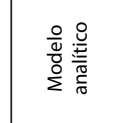 & 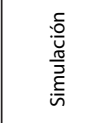 & 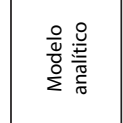 & 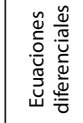 \\
\hline 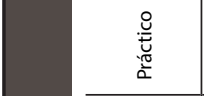 & 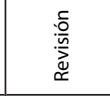 & 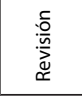 & 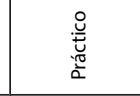 & 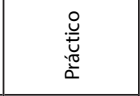 & $\begin{array}{l}\stackrel{8}{\overline{0}} \\
\stackrel{0}{\leftarrow}\end{array}$ & 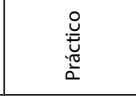 & $\begin{array}{l}\stackrel{8}{\bar{z}} \\
\stackrel{0}{\leftarrow}\end{array}$ & 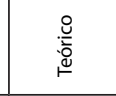 & 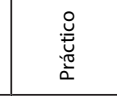 & 营 & 莺 & $\begin{array}{l}\stackrel{8}{\bar{z}} \\
\stackrel{0}{\circ}\end{array}$ \\
\hline 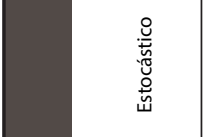 & $\underline{z}$ & $\underline{z}$ & 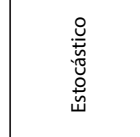 & 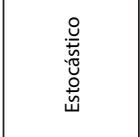 & 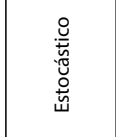 & 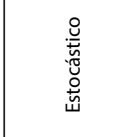 & 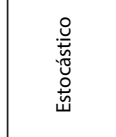 & 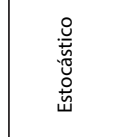 & 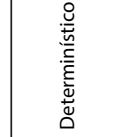 & 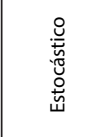 & $\begin{array}{l}\text { 总 } \\
\text { 莺 } \\
\text { 离 }\end{array}$ & 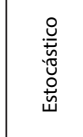 \\
\hline$\underline{z}$ & $\underline{z}$ & $\frac{\pi}{z}$ & $\frac{\pi}{z}$ & $\underline{z}$ & $\Sigma$ & $\frac{\circ}{\Sigma}$ & $\underline{z}$ & 气 & $\Sigma$ & $\frac{\pi}{z}$ & $\mathbb{z}$ & $\frac{\pi}{z}$ \\
\hline 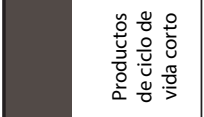 & $\underline{z}$ & $\underline{z}$ & $\Sigma$ & $\frac{\pi}{z}$ & 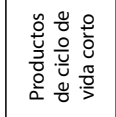 & $\frac{\pi}{z}$ & $\bar{z}$ & $\Sigma$ & 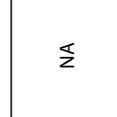 & $\frac{\pi}{z}$ & $\mathbb{z}$ & $\frac{s}{z}$ \\
\hline$\times$ & $\times$ & $\times$ & & & $\times$ & $\times$ & & & & & & \\
\hline & $\times$ & $\times$ & $\times$ & $\times$ & & $\times$ & $\times$ & & & & & \\
\hline$y$ & $x$ & $\times$ & $\times$ & $\times$ & & & & & & & & \\
\hline$\times$ & $\times$ & $\times$ & & $\times$ & & $\times$ & & $\times$ & $\times$ & $\times$ & $\times$ & $\times$ \\
\hline$\underline{\underline{v}}$ & $\times$ & $\times$ & $\times$ & & & $\times$ & $\times$ & & $\times$ & & $\times$ & \\
\hline 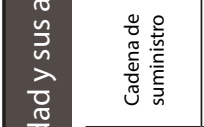 & 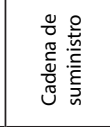 & 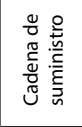 & 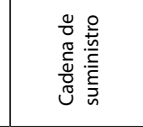 & 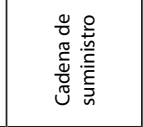 & 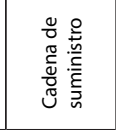 & 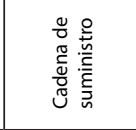 & 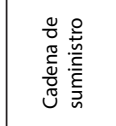 & 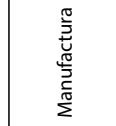 & 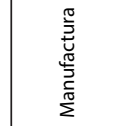 & 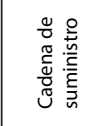 & 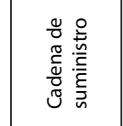 & 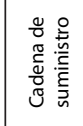 \\
\hline 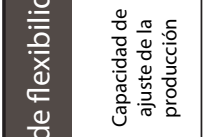 & 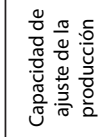 & 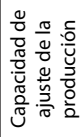 & 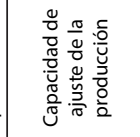 & 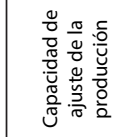 & 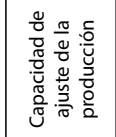 & 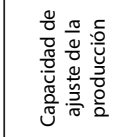 & 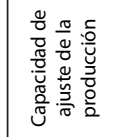 & 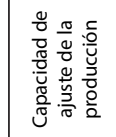 & 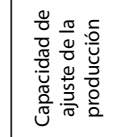 & 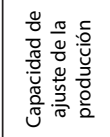 & 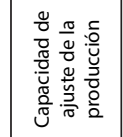 & 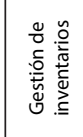 \\
\hline 㕝 & $\stackrel{i}{i}^{m}$ & 啇 & $\stackrel{\sim}{\sim}$ & $\bar{i}$ & 总 & స̃ & : & : & $\stackrel{m}{\stackrel{n}{a}}$ & ఫ్ & å & 高 \\
\hline 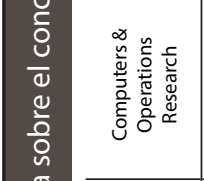 & 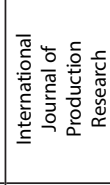 & 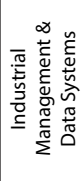 & 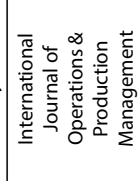 & 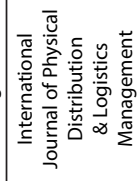 & 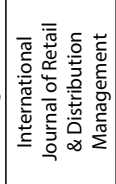 & 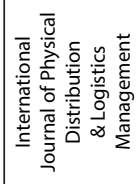 & 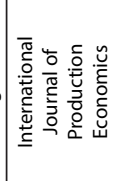 & 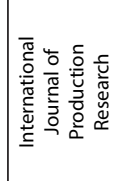 & 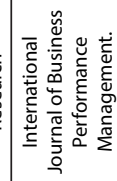 & 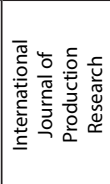 & 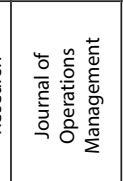 & 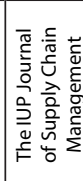 \\
\hline 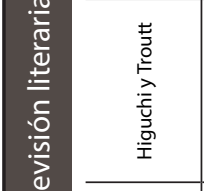 & 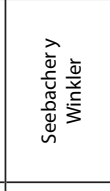 & 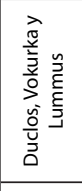 & 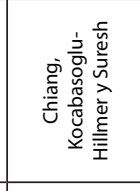 & 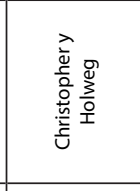 & 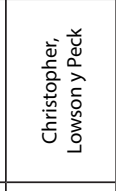 & 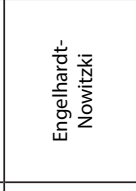 & 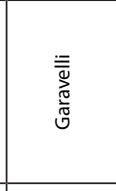 & 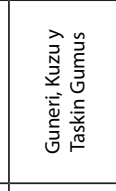 & 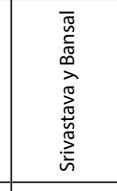 & 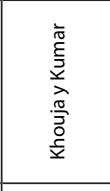 & 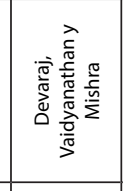 & 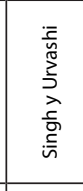 \\
\hline 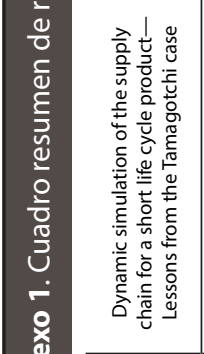 & 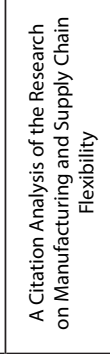 & 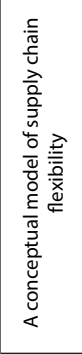 & 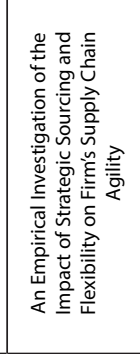 & 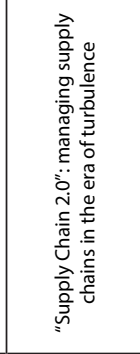 & 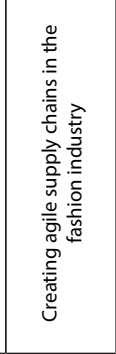 & 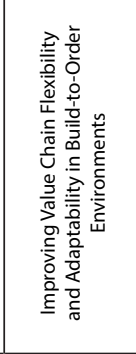 & 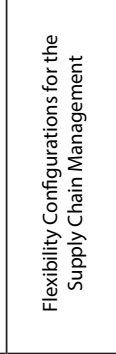 & 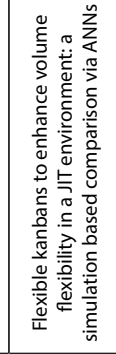 & 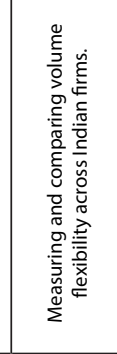 & 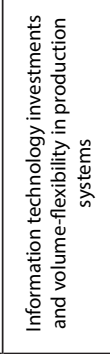 & 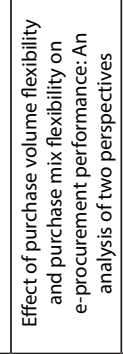 & 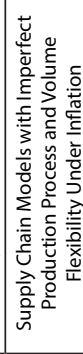 \\
\hline 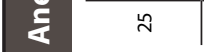 & $\stackrel{D}{\sim}$ & $\hat{\lambda}$ & $\stackrel{\infty}{\sim}$ & Nి & $\stackrel{\circ}{m}$ & $\bar{m}$ & $\tilde{m}$ & $m$ & $\stackrel{ \pm}{m}$ & $\stackrel{m}{m}$ & $\stackrel{\circ}{m}$ & $\hat{m}$ \\
\hline
\end{tabular}




\begin{tabular}{|c|c|c|c|c|c|c|c|c|c|c|c|c|}
\hline 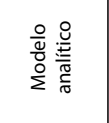 & 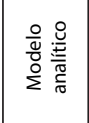 & 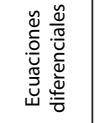 & 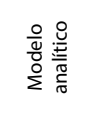 & 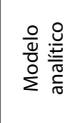 & 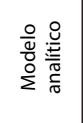 & 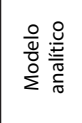 & 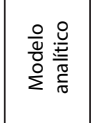 & 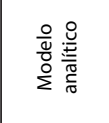 & $\Sigma$ & 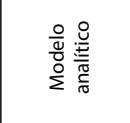 & 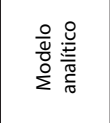 & 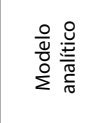 \\
\hline 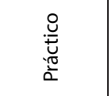 & 总 & $\begin{array}{l}\stackrel{8}{\overline{0}} \\
\stackrel{0}{\circ}\end{array}$ & 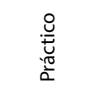 & 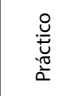 & 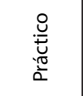 & 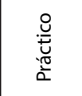 & 兽 & $\begin{array}{l}\stackrel{8}{\overline{0}} \\
\stackrel{0}{\circ}\end{array}$ & 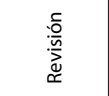 & 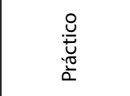 & 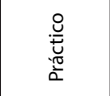 & 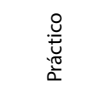 \\
\hline 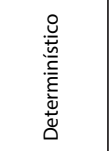 & 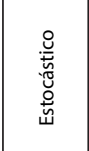 & 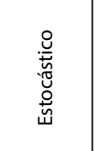 & 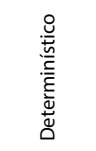 & 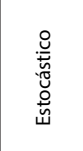 & 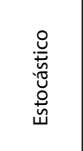 & 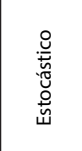 & 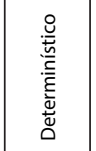 & 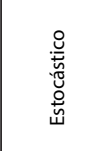 & $\Sigma$ & $\begin{array}{l}\stackrel{8}{*} \\
\text { 莺 } \\
\text { 离 }\end{array}$ & $\begin{array}{l}\stackrel{8}{*} \\
\text { 莺 } \\
\text { 离 }\end{array}$ & 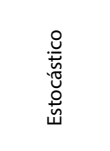 \\
\hline$\frac{\pi}{z}$ & $\frac{\pi}{z}$ & $\Sigma$ & $\underline{\underline{z}}$ & $\bar{z}$ & $\frac{\pi}{z}$ & $\bar{z}$ & $\frac{\pi}{z}$ & $\frac{\pi}{z}$ & $\stackrel{\pi}{z}$ & $\Sigma$ & 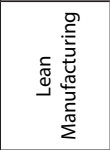 & $\underline{z}$ \\
\hline 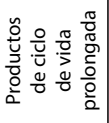 & $\underline{z}$ & $\Sigma$ & $\frac{\pi}{z}$ & $\frac{\pi}{z}$ & $\underline{z}$ & $\underline{z}$ & $\frac{\pi}{z}$ & $\underline{z}$ & 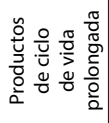 & $\frac{\pi}{z}$ & 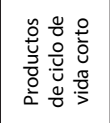 & 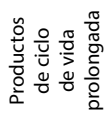 \\
\hline & & & & & & & & & $\times$ & & & $\times$ \\
\hline & & & & & & $\times$ & & $\times$ & $\times$ & $\times$ & $\times$ & \\
\hline$\times$ & & & & & & & & $x$ & $x$ & & & \\
\hline & $\times$ & $\times$ & $\times$ & $\times$ & $\times$ & $\times$ & $\times$ & $\times$ & $\times$ & $x$ & $x$ & $\times$ \\
\hline$\times$ & & $\times$ & & & & & $\times$ & $\times$ & $\times$ & & $\times$ & \\
\hline 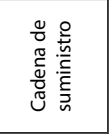 & 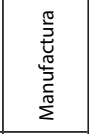 & 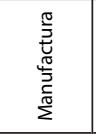 & 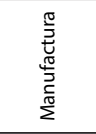 & : & 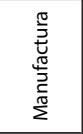 & 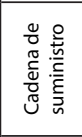 & 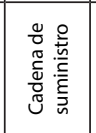 & 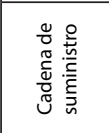 & 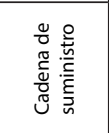 & 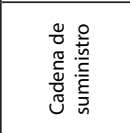 & 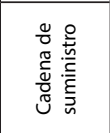 & 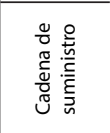 \\
\hline 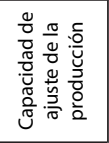 & 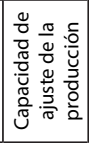 & 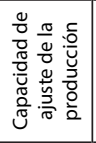 & 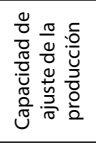 & 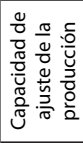 & 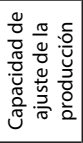 & 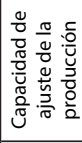 & 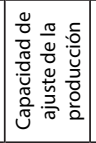 & 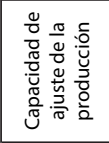 & 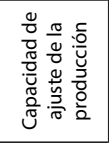 & 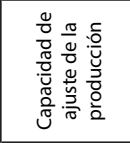 & 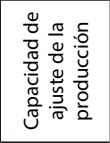 & 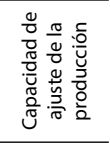 \\
\hline$\stackrel{+}{\stackrel{N}{N}}$ & $\stackrel{n}{\grave{d}}$ & $\overline{\bar{i}}$ & : & : & 蒿 & 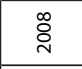 & $\stackrel{n}{\grave{N}}$ & $\stackrel{n}{\grave{N}}$ & $\stackrel{\text { }}{\sigma}$ & : & $\stackrel{\circ}{\circ}$ & $\overline{\bar{i}}$ \\
\hline 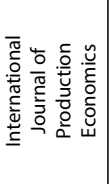 & 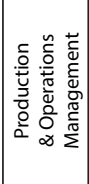 & 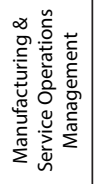 & 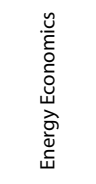 & 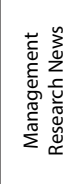 & 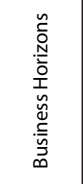 & 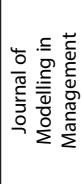 & 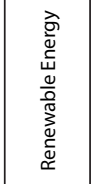 & 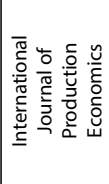 & 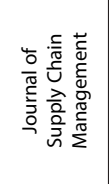 & 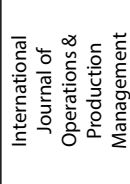 & 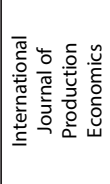 & 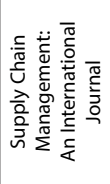 \\
\hline 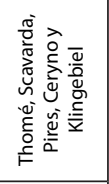 & 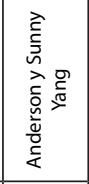 & 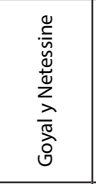 & 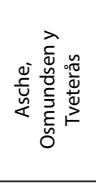 & 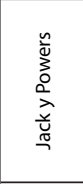 & 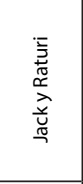 & 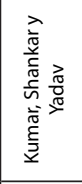 & 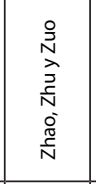 & 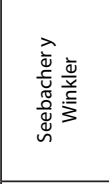 & 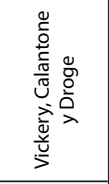 & 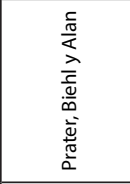 & 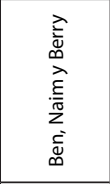 & 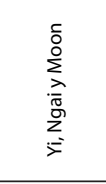 \\
\hline 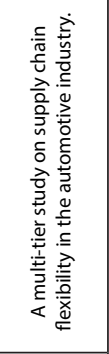 & 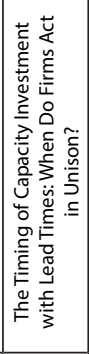 & 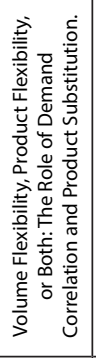 & 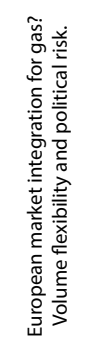 & 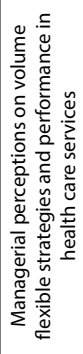 & 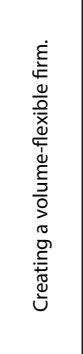 & 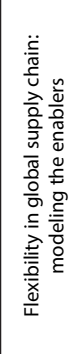 & 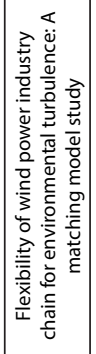 & 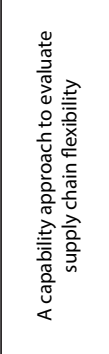 & 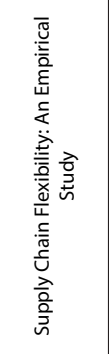 & 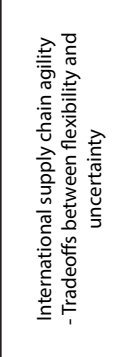 & 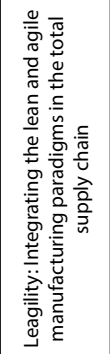 & 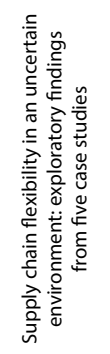 \\
\hline$\stackrel{\infty}{m}$ & \% & q & $\bar{f}$ & F & q & 7 & ஜ & \& & f & $\stackrel{\infty}{\sigma}$ & q & in \\
\hline
\end{tabular}

